\title{
The SED of the nearby $\mathrm{H}$ I-massive LIRG HIZOA J0836-43: from the NIR to the radio domain
}

\author{
Renée C. Kraan-Korteweg ${ }^{1}$ and Michelle E. Cluver $^{2} \dagger$, \\ ${ }^{1}$ Astronomy Department, Astrophysics, Cosmology and Gravity Centre (ACGC), University of \\ Cape Town, Rondebosch 7700, South Africa \\ email: kraan@ast.uct.ac.za \\ ${ }^{2}$ IPAC, California Institute of Technology, Pasadena, CA 91125, USA \\ email: mcluver@aao.gov.au
}

\begin{abstract}
HIZOA J0836-43 is one of the most H I-massive galaxies in the local $(z<0.1)$ Universe. Not only are such galaxies extremely rare, but this "coelacanth" galaxy exhibits characteristics - in particular its active, inside-out stellar disk-building - that appear more typical of past $(z \sim 1)$ star formation, when large gas fractions were more common. Unlike most local giant $\mathrm{H}$ I galaxies, it is actively star forming. Moreover, the strong infrared emission is not induced by a merger event or AGN, as is commonly found in other local LIRGs. The galaxy is suggestive of a scaled-up version of local spiral galaxies; its extended star formation activity likely being fueled by its large gas reservoir and, as such, can aid our understanding of star formation in systems expected to dominate at higher redshifts. The multi-wavelength imaging and spectroscopic observations that have led to these deductions will be presented. These include NIR $(J H K)$ and MIR (Spitzer; $3-24 \mu \mathrm{m}$ ) imaging and photometry, MIR spectroscopy, ATCA $\mathrm{H}$ I-interferometry and Mopra CO line emission observations. But no optical data, as the galaxy is heavily obscured due to its location in Vela behind the Milky Way.
\end{abstract}

Keywords. galaxies: individual (HIZOA J0836-43), galaxies: evolution, infrared: galaxies

\section{Introduction of HIZOA J0836-43}

The galaxy HIZOA J0836-43, originally discovered in the Parkes deep Multibeam H Isurvey of the Zone of Avoidance (ZOA; e.g. Kraan-Korteweg et al. 2005), is a rapidly rotating disk galaxy $\left(v_{\mathrm{rot}}=630 \mathrm{kms}^{-1} ; D_{\mathrm{HI}}=130 \mathrm{kpc}\right)$ with an H I-mass of $M_{\mathrm{HI}}=$ $7.5 \cdot 10^{10} \mathrm{M}_{\odot}$ and a dynamical mass of $M_{\mathrm{tot}}=1.4 \cdot 10^{12} \mathrm{M}_{\odot}$ (Donley et al. 2006$)$. It has a prominent bulge and smallish stellar disk $(B / D \sim 0.8)$ of evolved stars of $M^{*}=$ $4.4 \cdot 10^{10} \mathrm{M}_{\odot}$ embedded in the five times larger HI-disk, while its current hearty star forming activity makes it a luminous infrared galaxy (LIRG; next section).

HIZOA J0836-43 is the most H I-rich galaxy known. Such galaxies are extremely rare $\left(\sim 3 \cdot 10^{-8} / \mathrm{Mpc}^{3}\right)$ according to the current best determined local galaxy HI-mass function (HIMF; Zwaan et al. 2005) and should not have been found at all in the explored volume. Interestingly, the recent, larger volume ALFALFA survey 40\%-data release (Haynes et al. 2011) identify several, similarly extreme H I-massive galaxies in excess of the predicted HIMF number density. Independently, galaxies with such vast reservoirs of gas were more common in the past, as were LIRGs. Hence this enigmatic, relatively nearby $(v=$ $10689 \mathrm{~km} \mathrm{~s}^{-1}$ ) galaxy is an ideal local probe that enables detailed studies of evolutionary processes and the transformation of gas into stars.

$\dagger$ Present address: AAO, Epping NSW 1710, Australia 


\section{The Observational Data}

The galaxy lies deep in the ZOA $\left(\ell=262^{\circ} .48, b=-1.64\right)$ and suffers from severe Galactic foreground extinction $\left(A_{V}=7.3\right)$, rendering it practically invisible in the optical. Hence our detailed follow-up observations focus on the infrared, radio and $\mathrm{mm}$ domain.

IRSF NIR imaging survey. The deep JHK-imaging survey of $2.2 \square^{\circ}$ with the Infrared Survey Facility (IRSF) in Sutherland (SAAO) aimed at learning more about an environment that permits such a gas giant to evolve. The survey uncovered 404 galaxies to the completeness limit of $K_{s}<15$. 8 (Cluver 2009). It finds the volume around HIZOA J0836-43 to be overdense in sub- $L^{*}$ systems, and none in the $L^{*}$ range. Hence it seems to live in a tranquil, underdense environment on the edge of a void. There are no indications of a major merger that could have triggered a starburst (see also Fig. 2). This may have allowed the formation of its giant $\mathrm{H}$ I-disk through minor merger or accretion - and aided its survival.

Spitzer IRAC and MIPS imaging and spectroscopy. The photometry from the Spitzer IRAC and MIPS band was presented in Cluver et al. 2008. It is reproduced in the SED (Fig. 1) together with the IRSF data, the DENIS I-band (Donley et al. 2006) and, now available, WISE $12 \& 22 \mu \mathrm{m}$ fluxes (Jarrett, priv. comm.).

The resulting total infrared luminosity $L_{\mathrm{TIR}}=1.2 \cdot 10^{11} \mathrm{~L}_{\odot}$ defines it as a LIRG. Despite the high star formation rate $\left(\mathrm{SFR}=20.5 \mathrm{M}_{\odot} / \mathrm{yr}\right)$, the comparison of its $\mathrm{SED}$ to various galaxy model templates, and the archetypal local starburst M82 in Fig. 1, shows that HIZOA J0836-43 is neither a starburst galaxy nor - as originally assumed from its NIR morphology - a S0/Sa-galaxy. It rather resembles a Sc galaxy with strong emission in the mid-IR $5-20 \mu \mathrm{m}$ range, due to the prevalence of PAH molecules, and a prominent FIR cold dust continuum. The latter is substantiated by the mid-IR spectroscopy (Cluver et al. 2010) which shows particularly luminous 6.2 and $7.7 \mu \mathrm{m}$ PAH features (stronger than typical starbursts; e.g. Brandl et al. 2006) in contrast to the continuum emission from warm dust. The latter is surprisingly weak (i.e. lower than any of the local LIRGs in GOALS), suggestive of an extended dust geometry. Cold dust is plentiful, however,

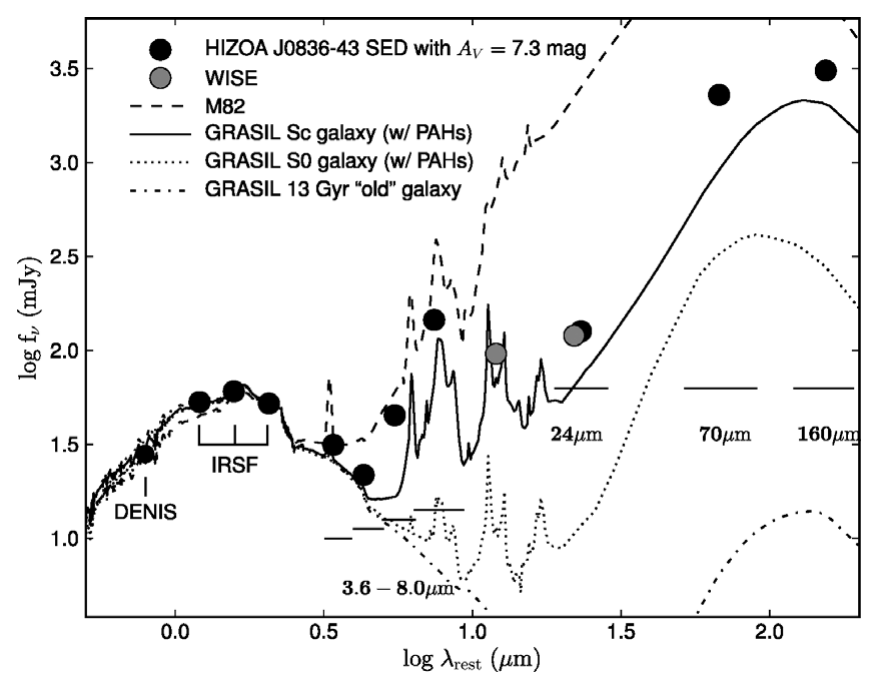

Figure 1. SED based on DENIS $I$, IRSF $J, H, K_{s}$, Spitzer IRAC $(3.6-8.0 \mu \mathrm{m})$ and MIPS $(24$, $70 \& 160 \mu \mathrm{m})$, as well as the WISE $12 \& 22 \mu \mathrm{m}$ bands - all corrected for extinction $\left(A_{V}=7^{\mathrm{m}} \cdot 3\right)$. Superimposed are GRASIL model templates and the M82 spectrum for comparison. While the high MIR/FIR luminosity makes this galaxy a LIRG, it clearly is not consistent with a starburst. (Adapted from Cluver et al. 2008). 
and a dominant contributor to the total infrared luminosity with its steeply rising FIR emission for $\lambda \gtrsim 60 \mu \mathrm{m}$.

The IRS spectroscopy of the nucleus further reveals strong excited nebular lines (e.g. [NeII], [S III], [Si II], [Ar II]). While these are typical of starburst regions, the line ratios indicate a softer radiation field (like PDR's) and AGN activity is weak or absent. This is independently confirmed by the absence of [Ne V] and [O IV] lines.

ATCA and MOPRA observations. With the surprising dearth of warm dust despite the strong star forming activity (PAH's) and the large reservoir of cold H I gas, Mopra CO line observations (1-0) were used to probe the cold molecular gas content. The spectrum shows a double horn signal, matching the $\mathrm{H}$ I-profile width perfectly. However, the total molecular gas mass $\left(\mathrm{H}_{2}+\mathrm{He}\right)$ of $3.9 \cdot 10^{9} \mathrm{M}_{\odot}$ is lower by a factor of three than expected from the infrared luminosity, and only a meager $5 \%$ compared to the atomic H I (Cluver et al. 2010). Both the total and cold molecular gas fraction (gas/gas+stellar mass) are high $(64 \%$ and $8 \%$ ) compared to the mean of local galaxies (e.g. $20 \%$ total; Leroy et al. 2008, and $6 \%$ molecular for the CO-detected stellar-selected massive galaxies; Saintonge et al. 2011), but low compared to the local LIRG sample (Wang et al. 2006). Such high gas fraction values are the standard though at higher redshifts (e.g. Daddi et al. 2010 for their NIR selected $B z K$ sample at $z \sim 1.5$ ).

Given this dichotomy, it seemed of importance to learn more about the interplay between star formation activity and gas physics in the galaxy itself. This required longer baseline observations with ATCA to complement the earlier Donley et al. (2006) data, which only had $30^{\prime \prime}$ angular resolution. Of interest is the connection - if any - with the star forming region, which extends beyond the old stellar bulge, suggestive of inside-out stellar disk-building, and exhibits a warp towards the eastern side. This is evident in the composite NIR \& MIR image (left panel of Fig. 2) as well as the composite spectral map ( $2^{\prime \prime}$ resolution) of the PAH 6.2, 7.7 and $11.3 \mu \mathrm{m}$ features (right panel). Note that the latter was obtained from SL $(5-14 \mu \mathrm{m})$ IRS observations mapping across the IR disk.

A preliminary H I-map from the newly acquired data is superimposed on the right panel in Fig. 2 for comparison. We note that the improved spatial resolution reveals an enhancement in the atomic gas exactly at the location of the warp. This may be the result of a recent tidal interaction which has triggered star formation as gas flows towards the center of the galaxy. This H I-data will be explored in detail in Cluver et al. (in prep).
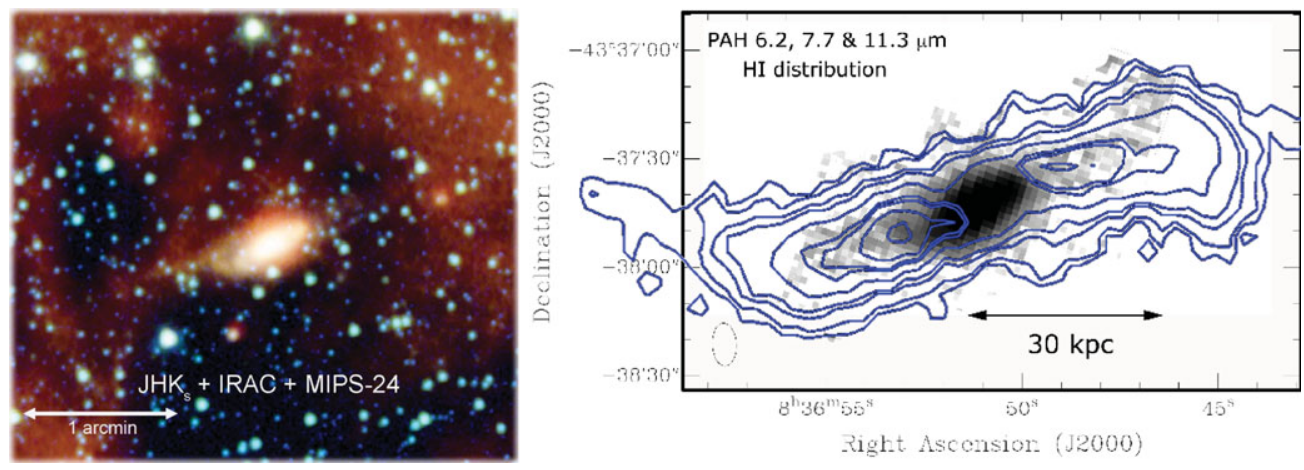

Figure 2. Left Panel: Composite of $J, H, K_{s}$ and Spitzer IRAC \& MIPS $24 \mu \mathrm{m}$ images. Right panel: H I distribution superimposed on composite spectral maps of the $6.2,7.7$, and $11.3 \mu \mathrm{m}$ PAH emission bands from Spitzer IRS. Note the asymmetry of PAH emission on the left (beyond the edge of the stellar disk), coinciding with an enhancement seen in the H I. 


\section{Connection to evolutionary processes at higher redshifts}

How does this galaxy relate to systems at varying redshift/evolutionary stages? Albeit at the extreme end, HIZOA J0836-43 seems to fall exactly on the H I-mass and SFR relation defined by the nearby SINGS galaxy sample (contrary to M82 or Malin 1). This suggests it to be a scaled-up version of local disk galaxies. Comparing it to the local LIRG sample $\left(z \lesssim 0.1\right.$; Wang et al. 2006) its specific starformation rate of $0.47 \mathrm{Gyr}^{-1}$ appears to follow the overall trend. But, it again lies at an extreme stage, implying active stellar building; in an additional 2 Gyrs of time it will double its stellar mass and fit the 'current' local LIRG data. This hint of relic SF properties is also seen when comparing HIZOA J0836-43 to the higher redshift SF galaxy sample by e.g. Bell et al.(2005), where it fits well on sSFR-relation of $z \sim 0.7$ galaxies, but does not conform at all to the local SF galaxy sample. Idem ditto when compared to the Genzel et al. (2010) sample where it closely aligns with the $z \sim 1.2$ sample. Tellingly, when entering the parameters of HIZOA J0836-43 in the so-called main sequence of SFR as a function of stellar mass and redshift (Bouché et al.2010) a redshift (age) of $z \sim 0.95$ is returned, suggesting a tantalising link to a distant epoch of star formation.

HIZOA J0836-43 displays a combination of high gas fraction with low efficiency, but extended, star formation in a low excitation disk, also found in the sample of normal, near-IR selected $(B z K)$ galaxies at $z \sim 1.5$ of Daddi et al. (2010), who consider them scaled-up local disk galaxies. Since larger gas fractions at higher redshift permit larger $L_{\mathrm{IR}}$ before invoking special events like mergers that show an offset in correlations with $L_{\mathrm{IR}}$ (Nordon et al. 2011), HIZOA J0836-43 can be studied as an analogue of disk building processes dominating at the peak of stellar mass growth.

The lower fraction of molecular gas in HIZOA J0836-43 compared to detected highredshift galaxies (Tacconi et al. 2010) highlights the need for an accurate molecular gas inventory, in parallel to increased high $z$ detections. Though, as pointed out by Groves at this conference, "dark" molecular gas may be a feature of the active ISM enrichment occurring in the disk. Herschel and ALMA is needed to provide a cohesive picture that can be used to understand disk building in gas-rich systems.

\section{Acknowledgements}

RKK thanks the South African National Research Foundation and the University of Cape Town for financial support.

\section{References}

Bell, E. F., Papovich, C., Wolf, C., Le Floc'h, E. et al. 2005, ApJ, 625, 23

Bouché, N., Dekel, A., Genzel, R., Genel, S. et al. 2010, ApJ, 718, 1001

Brandl, B. R., Bernard-Salas, J., Spoon, H. W. W., Devost, D. et al. 2006, ApJ, 653, 1129

Cluver, M. E. 2009, PhD thesis, University of Cape Town

Cluver, M. E., Jarrett, T. H., Appleton, P. N., Kraan-Korteweg, R. C., et al. 2008, ApJL, 686, L17

Cluver, M. E., Jarrett, T. H., Kraan-Korteweg, R. C., Koribalski, B. et al. 2010, ApJ, 725, 1550

Daddi, E., Elbaz, D., Walter, F., Bournaud, F. et al. 2010, ApJ, 714, L118

Donley, J., Staveley-Smith, L., Koribalski, B. et al., 2006, MNRAS, 369, 1741

Haynes, M. P., Giovanelli, R., Martin, A. M., Hess, K. M. et al. 2011, AJ, 142, 170

Genzel, R., Tacconi, L. J., Gracia-Carpio, J., Sternberg, A. et al. 2010, MNRAS, 407, 2091

Kraan-Korteweg, R. C., Staveley-Smith, L., Donley, J. et al. 2004, IAU Symp. 216, 203

Leroy, A. K., Walter, F., Brinks, E., Bigiel, F., \& de Blok, W. J. G. 2008, AJ, 136, 2782

Nordon, R., Lutz, D., Berta, S., Wuyts, S. et al. 2011, arXiv:1106.1186

Saintonge, A., Kauffmann, G., Kramer, C., Tacconi, L. J. et al. 2011, MNRAS, 415, 32 
Tacconi, L. J., Genzel, R., Neri, R., Cox, P. et al. 2010, Nature, 463, 781

Wang, J. L., Xia, X. Y., Mao, S., Cao, C. et al. 2006, ApJ, 649, 722

Zwaan, M. A., Meyer, M. J., Staveley-Smith, L., \& Webster, R. L. 2005, MNRAS, 359, L30

\section{Discussion}

Chakrabarti: What is your interpretation of the high IR emission? My interpretation is that this is due to a minor merger. We now have scaling relations that will allow you to derive the satellite mass from the H I-map. So I suggest that you calculate the Fourier amplitude.

KraAn-KorteweG: Thank you. That is an interesting idea for the future, but we will await the reduction of our new $\mathrm{H}$ I-data and its analysis first. The current $\mathrm{H}$ I-data maps are quite smooth over most of its gas disk without any kinks in its contours. This makes the minor-merger scenario unlikely; at least, it could not have occurred over the last 2 Gyr or so to account for such a stable disk. Secondly, while a minor merger (or instability caused by the accretion of a satellite) might explain the strong IR luminosity due to triggered star formation/nuclear starburst, it does not account for the extreme mass in H I-gas in HIZOA J0836-43, nor the abnormally high gas fraction. In addition, the latter scales beautifully with normal - albeit higher redshift - starforming galaxies. What remains unresolved as yet is the question, how HIZOA J0836-43 could have acquired/accreted all this cold gas.

Bureau: The comparison of the $\mathrm{CO}$ and $\mathrm{H}$ in the spectra shows a lack of $\mathrm{CO}$ (thus $\mathrm{H}_{2}$ ) in the rising part of the rotation curve, hence central part of the galaxy. How can this be reconciled with signatures of star formation in the centre, e.g. in the nuclear infrared spectrum shown.

KRAAN-KORTEWEG: Note that the "nuclear spectrum" covers the "nuclear region" and is a 9.25 extraction, so it picks up more than just the nucleus itself. Secondly, the central low-velocity gas might well be present at a lower level compared to the H I-gas distribution (i.e. not detectable with the current sensitivity of our Mopra observations). This is seen quite often in other spiral galaxies when comparing $\mathrm{HI}$ and $\mathrm{CO}$ observations. Much of the very central CO may also have been used in a previous epoch of star formation which produced the current old stellar bulge (which has a disk scale length of $4 \mathrm{kpc}$, respectively $\left.7^{\prime \prime}\right)$. But interesting is that the steep rotation in the $\mathrm{H}$ I velocity appears matched to the $\mathrm{CO}$, so the central region achieves a high velocity close in. This may be important for extended building into the disk. We really need spatial information accompanied by velocity resolution (as we now have with $\mathrm{HI}$ ) to understand this, and ALMA will make this possible. Also, given the prominence of [Si II] in the spectrum, it is not inconceivable that some of the $\mathrm{CO}$ is being photodissociated, whereas the molecular hydrogen is shielded, making it "dark". Herschel (proposal submitted) in combination with ALMA (planned) will enable a detailed study of such a mechanism. 